it is suggested that the impact of the Conference Abstracts would be strengthened by providing more space to one (or more) of the invited lecturers for a complete version of their highly informative papers. In any event the abstracts of invited lectures should contain the leading references.

Kirste (Mainz) and Schelten (Jülich), who were among the first to apply neutron scattering to an elucidation of the near order structure of polymers, talked about their subjects, Kirste treating amorphous materials, particularly the beginning of phase separation in blends of polystyrene-co-acrylonitriles (PSAN) of different AN content and Schelten problems of the "adjacent re-entry" in semicrystalline polymers (experimental errors of scattering data, nitric acid etching to confirm escape of molecules from a crystallite, comparison with Flory-Yoon calculations). The presentation of Schelten and later talks from Mainz and Bristol have shown that three groups presently working on the conformation of chains in semicrystalline polymers are now approaching identical conclusions; that is, essentially re-entrant chain folding in single crystals grown from solution, and no regular folding in the rapidly crystallized melt. This agreement seems to be one of the main achievements of the meeting owing to the rather controversial nature of this topic.

Then came Carpenter (Chicago) who reported on new instrumental developments. Pulsed neutron sources used with large area detectors widen the range of wave lengths $\lambda$ available which are sorted by time-of-flight diffractometers with $\Delta \lambda / \lambda<0.01$.
Contributed papers dealt, for example, with the conformation of $n$-alkane molecules in the melt and in solution, with local orientation correlation in partially crystallized polymers (also studied by the anisotropy of thermal diffusivity), with "reptational motions" of diffusing molecules (corroborated by micro-IR measurements), and with the conformation of chains in drawn polystyrene (using also partially labelled chains). The latter experiments confirmed an affine deformation of the average chain end-to-end vectors but revealed a degree of orientation for the molecular segments, which deviated the more from an affine deformation the higher the arawing temperature.

H.H. Kausch (EPFL, Lausanne)

\title{
- ECOSS I - Europe Comes Together on Surface Science
}

Surface Science is not only dynamic and rapidly developing, but might even be considered to be growing mature ; so mature in fact, that it is now appropriate to replace the several biennial, quasi-international conferences by a joint annual European Conference on Surface Science. The first, organized under the auspices of EPS, IUPAP, and IUVSTA, was held in Amsterdam, 5-9 June, 1978.

Progress as reported at this meeting is, indeed, taking place on a very wide front. This reflects the breadth of the field, which could be defined as either "multidisciplinary, interdisciplinary or as a sub-discipline on its own " 1 ). The Surface attracts physicists, chemists, and technologists, and within physics alone, contributions are made by people with a great variety of background - from atomic, molecular, and solid-state physics. This makes the picking-out of conference highlights difficult and a matter of personal interest.

The Conference gave me the definite impression that we have now gone a long way towards experimentally characterizing the atomic and electronic structures of surfaces, and that it is becoming more and more proper to ask questions about how to use the increasingly detailed information thus obtained. This leads us to the core of Surface Science ; issues that earlier were fashionable essentially as research proposals (corrosion, heterogeneous catalysis, ...) are today, subjects for active surface-science research.

Judging from the Conference $\mathrm{Ab}$ stracts ${ }^{2}$ ), structure, electron properties, chemisorption and catalysis should be the major fields of interest right now. Taking this in conjunction with the impression gained at last year's international conference in Vienna, one sees a clear trend of methods becoming established for the determination of atomic and electronic structure of surfaces and a strongly growing interest in surface reactions.

The Nestor among the structuredetermining methods, low-energyelectron diffraction (LEED) by now has revealed almost 100 surface structures, and one now dares to talk about surface crystallography as a discipline. Such a term would denote a wide area and include many other techniques. For instance, new medium energy-ion-scattering data were reported, which resolve the position of a sulphur atom on a nickel surface with a claimed accuracy of $0.01 \AA$. Methods utilizing $X$-rays for excitation and diffracted Auger- or photoelectrons for detection seem to have got off the ground, and angularresolved photoemission in the ultraviolet and electron-energy-loss spectroscopy are also giving valuable supplementary information about, in particular, sites of adsorbed species. The overwhelming majority of the structural data is still nevertheless coming from LEED, with a claimed accuracy of about 0.05-0.01 § perpendicular, and $0.2 \AA$ parallel to the surface. The great progress made during the past years now makes it possible to obtain a coherent picture of, e.g., certain adsorbate-substrate combinations.

The study of the electron structure is dominated by photoelectron spectroscopy. Angular resolution, in particular when used with polarized synchrotron radiation in the ultraviolet, permits the direct determination of the dispersion curves for surface and bulk states, as well as of band/ adsorbate orbital symmetries. For chemisorption and surface reactions it gives information about ionization potentials and provides "finger-prints" of adsorbed species. Ultraviolet photoemission can now give electron energies with an accuracy that should be provoking for the theorists.

Another problem that occupies several theorists, concerns shake-up and relaxation processes occuring upon photoexcitation of core electrons in adsorbed species. There is now some understanding of the additional structures that appear in the adsorbate core level photoelectron spectra and which are not present in the corresponding spectra of atoms and molecules in the gas phase.

The long list of surface-reaction papers clearly indicates that progress has been made at a rapid rate in the fields of oxidation, adsorption of molecules - not just the favourite proto- 
type $\mathrm{CO}$ but a whole range of small and big molecules -, and catalytic reactions. The whole spectrum of surface diagnostic tools seems to be used to probe primarily the end products of the reactions or possible intermediates. There were also reports on processes that give information about the early stages of reactions, for instance surface chemiluminescence.

Invited talks and contributed papers on catalysis, the insulator-semiconductor interface, metallurgy, friction, and lubrication gave an indication of the central role played by Surface Science.

When looking at the abstracts and posters or listening to the talks, the symptoms of a creeping disease of Surface Science are seen: LEED, MEED, RHEED, SIMS, AES, XPS, HRELS, UP, TPTD, ESCA, TEM, ESDIAD, ISS, SXAPS, EELS, ARUPS, SRPS, SPLEED, FEM, TD, ESD, DTA, ISS, SAM ,FEED, MBE, RED, SEM, SCANIIR, BLE, MEIS, SES, MEM, LID,
LEIS, RESLEET, INS, ..... Some people claim that these symbols stand for useful experimental techniques, and others say things like " ... the EDC from UPS under UHV relates to the DOS." The question is whether we should and could stop the exponential growth in these "four letter" words.

Statistics about Surface Science in Europe ') gathered from this and recent conferences and the journal Surface Science, indicate amongst other things - an under-representation from eastern Europe, with however a positive gradient, and a decrease in contributions from industrial laboratories. This last should be seen as a warning for future annual meetings of the ECOSS series, especially as the coordination aims have been expressed as : "... to serve the needs and aspirations of European research workers in the field of Surface Science and to promote their communication, $\ldots$ and to ensure a high scientific standard, the interdisciplinary nature of the field and the inclusion of new developments in both fundamental and applied areas "1).

The next opportunities to work towards these aims will be at

- ECOSS II in Cambridge, England, 26-29 March, 1979

- ECOSS III in Cannes, France, 2127 September, 1980

- ECOSS IV in Germany, 1981.

The "supermarkets with posters" ") of ECOSS I might then be partly abandoned, whilst still avoiding parallel sessions. Surface Science is in too rapid a state of development to allow divisions into fixed branches. Crossfertilization is a MUST in this active field.

\section{Footnotes :}

1. Welcome remarks by G.A. Bootsma, President of the Nederlandse Vacuumvereiniging.

2. ECOSS I, Book of Abstracts, in Nederlands Tijdschrift voor Vacuum Techniek, 16e, Jaargang Nr. 2/3/4 (1978).

B. Lundqvist

(University of Aarhus)

\section{0th European Conference on Atomic Spectroscopy}

As usual, the annual meeting of the "European Group for Atomic Spectroscopy (EGAS)" took place in the second week of July. Attended by 264 spectroscopists from 19 countries, both West and East, splendidly organized at the Garching Research Centre by $\mathrm{H}$. Walther and his colleagues from the Physics Department of the University of Munich and the Max-PlanckInstitut for Plasmaphysics at Garching, this was a fitting occasion for the 10th anniversary. EGAS, a Section of the Atomic Physics Division of EPS, in its development has, from the beginning, kept a style of its own. When founded at Caen (Normandy) its main aims were formulated as being to :

(i) bring together regularly European atomic spectroscopists in order to foster the exchange of results and ideas and, different from many other societies and groups.

(ii) give especially the younger colleagues a chance - a theme constantly repeated.

One main trend of present scientific work is that the laser seems no longer to be mainly an object of research per se, but rather has become a research tool for obtaining results in atomic, nuclear and also fundamental physics. Here W. Demtröder reviewed the latest state of high resolution or even sub-Doppler spectroscopy and the more sensitive detection techniques as, e.g., saturation interference spectroscopy within a Jamin-Interferometer.

For one of these newer methods "Fast Ion Beam Laser Spectroscopy," where the Doppler width is reduced by " acceleration cooling," the basic principles, essential requirements and significance were dealt with in detail by M.L. Gaillard and in a number of contributed papers. For the future, important results may be expected for high resolution spectroscopy of atoms and molecules, for collisions in excited states, and for hyperfinestructure (hfs) research in "exotic" atoms.

The importance of the laser as a tool became further manifest by the fact that many - also more conventional - applications of lasers were reported in a large number of sessions and papers. They were concerned, e.g., with hfs and isotope shift (IS) investigations, lifetime measurements, the search for the first optical transition in francium or the determination of the fine structure (FS) separation of high Rydberg states by quantum beats (applying an electric field for the detection).
For such highly excited atoms in electric fields S. Feneuille reviewed the theoretical treatment and experimental facts much of which can be understood only recently.

A further new way of experimenting was also sketched : it connects atomic and nuclear physics beyond the traditional coupling through shell-nucleus interaction (hfs and IS). As demonstrated by E.W. Otten and his group impressive results on the nucleus (e.g. $\delta<r^{2}>$ ) can be obtained by combining optical pumping with nuclear research techniques ( $\beta$-decay). Thus systematic hfs and IS investigations were possible of several shortlived isotopes with the laser in line with an ion beam, as in ISOLDE at CERN.

In future, laser applications may also be decisive for the fundamental question of parity violation in atomic physics. Mme M.A. Bouchiat, (one of the originators of research into parity non-conserving effects in atomic physics) reviewed the present confusing situation. Of three experiments to measure the optical rotation of $\mathrm{Bi}$ atoms (Oxford, Seattle, Novosibirsk) only in the Soviet experiment were the rotations said to be consistent with theory. On the other hand, in a 